\section{Living images in print}

\section{David Gadian \& Graeme Bydder}

Reviews of Magnetic Resonance in Medicine. Editor-in-chief John C. Gore. Pergamon. 2/yr. £60, \$100 (institutional); individual rates also available.

Journal of Medical Imaging. Editor-inchief H.E. Schütte. Elsevier. 6/yr. Dfl 325, $\$ 158.50$.

IT Is only three years since two new journals dealing with aspects of magnetic resonance in medicine were reviewed here (Nature 317, 299; 1985). Now we have two others in a similar or related area. Are there too many? Possibly, but this is still a rapidly growing field.

Ultimately, the success of any journal is determined by the quality of its contents. This is particularly true for such a publication as Reviews of Magnetic Resonance in Medicine, for invited reviews may be uneven in quality and usefulness, and may appeal to very different audiences. The first three issues of this journal contain

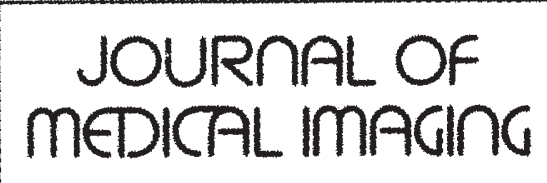

just seven articles, taking about 350 pages, and although they do show some variability some excellent work has been published. Thus although the very first review (by K. Wuthrich), on the structure and function of proteins and nucleic acids, may appear to be on the fringe of applications to medicine, it was appropriate to begin with a review of this subject, especially because NMR techniques that are initially developed for structural studies may subsequently prove valuable in imaging or metabolic studies. Particularly noteworthy articles, more directly related to in vivo studies, have been on localization methods for in vivo NMR spectroscopy (W.P. Aue) and on artefacts in magnetic resonance imaging (R.M. Henkelman and M.J. Bronskill).

We understand that publication is somewhat behind schedule, but the contributions remain up to date. For example, that by Henkelman and Bronskill (first issue of 1987) contains many references to work published in 1987. Reviews covers all aspects of magnetic resonance in medicine, and although papers are generally invited, there is also scope for authors to submit contributions. Here is a journal that shows considerable promise.

Journal of Medical Imaging covers clinical aspects of radiology and medical imaging in the broadest sense, including

Enzymes that work

Christopher R. Lowe

Biocatalysis. Managing editor David Best. Harwood. 4/volume. UK £164, North America $\$ 268$, elsewhere $£ 204$ (corporate); UK 1110 , North America $\$ 182$, elsewhere $£ 138$ (library); UK £56, North America $\$ 90$, elsewhere $£ 70$ (personal).

THE information explosion in biotechnology threatens to overwhelm even the most ardent of readers among us. Any new journal, therefore, stands a good chance of being criticized as simply adding to the myriad of pseudo-biotechnology publications already in the market place and fuelling the competition for that scarcest of all resources, the quality manuscript.

The managing editor of Biocatalysis is clearly sensitive to these issues and has, quite sensibly, focused his journal specifically on the underlying science and potential industrial exploitation of biological catalysts for the interconversion of chemical species. The advent of the journal is timely, because of the intense worldwide interest in biotransformations directed towards the production of new synthetic routes.

The topics covered include the regioand stereospecificity of biological catalysts, the reaction conditions under which they operate, the development of biocatalytic systems from discovery to commercialization, the integration of chemical and biological processes to produce novel synthetic routes, and the engineering aspects of the industrial implementation of these systems.

Inevitably, the scope of Biocatalysis overlaps somewhat with that of both established and newly introduced journals, notably Enzyme and Microbial Technology, Journal of Chemical Technology and Biotechnology and Applied Biochemistry and Biotechnology, but there is much to be said for having a choice of journals in an actively expanding area of interdisciplinary research. The declared intention of Biocatalysis, to act as a focal point for chemists, biochemists, microbiologists, chemical engineers and biochemical engineers, is soundly based.

The journal has an unmistakable international flavour, with a well-balanced selection of regional editors and members of the editorial board. Each issue has contained five to nine articles, some of them short reviews, some primary research papers. Not unexpectedly, many of the contributions published to date have originated from members of the editorial board, but these are distinguished authors and their papers have been of a consistently high quality. A welcome feature is the grouping of the solicited reviews into 'themes', the first series being collectively entitled "Biocatalysis in Multiphase Environments". The format of the journal is easy and readable, and (much to my personal delight) the titles of work cited are included in the references.

In all, Biocatalysis should appeal to a substantial body of readers involved in applied enzymology. Those self-same readers, however, might well also be interested in other aspects of the 'applied' literature, most notably that dealing with patents, and the editors might consider including some review of this literature, perhaps to appear in the thematic issues. But this mild criticism should not deter readers from subscribing to what may become a very successful journal.

Christopher R. Lowe is Director of the Institute of Biotechnology, University of Cambridge, Downing Street, Cambridge CB2 $3 E F, U K$.

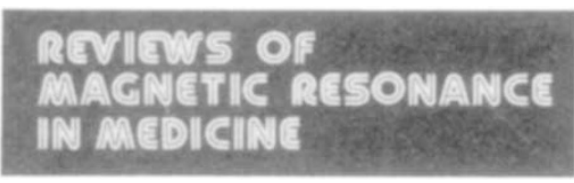

nuclear medicine, ultrasound and magnetic resonance imaging. The journal contains review articles, descriptions of new techniques, research papers and case reports. The delay between submission and publication in Vol. 2 is typically 5-12 months, allowing for revision in most cases. Although this is the official publication of the Netherlands Society of Radiodiagnosis, it has attracted contributions from Hong Kong, Japan, the United States and many European countries other than the Netherlands. Interestingly, in the advertisements Toshiba offers products conducive to the Dutch 'budget unfriendly' climate.

There are now over 60 radiology journals, and Journal of Medical Imaging is unusual among the newer ones in that it covers the entire subject - with the exception of the European Journal of Radiology, journals that have appeared over the past ten years have specialized in topics such as ultrasound, computed tomography or magnetic resonance imaging. So the newcomer will compete with much larger and better established publications. It will probably achieve a respected position among European general radiological journals alongside the likes of Clinical Radiology, to which it is most similar in scope and aims.

David Gadian is Rank Professor of Physics in Relation to Surgery at the Hunterian Institute, Royal College of Surgeons of England, Lincoln's Inn Fields, London WC2A 3PN, UK. Graeme Bydder is Senior Lecturer in the NMR Unit at Hammersmith Hospital, Ducane Road. London W12 OHS, UK. 